\title{
Colojejunal Fistula Resulting from a D-PEJ Feeding Tube
}

\author{
Martin D. Zielinski Robert R. Cima \\ Department of Colon and Rectal Surgery, Mayo Clinic College of Medicine, \\ Rochester, Minn., USA
}

\section{Key Words}

Colojejunal fistula · Complications · D-PEJ · Feeding tube

\begin{abstract}
Numerous procedures have been developed to provide adequate enteral nutrition to patients with gastrointestinal disorders. Previously, operative placement of a feeding gastrostomy or jejunostomy tube was the accepted means of gaining chronic enteral access. However, improved technology and experience with endoscopic techniques have quickly replaced primary operative placement of enteral access. Direct percutaneous endoscopic jejunostomy (D-PEJ) is a procedure that was designed to deliver enteral feeding solutions for patients with proximal disease after unsatisfactory results from percutaneous endoscopic gastrostomy tubes with jejunal extensions (PEG-J). As with any procedure, it is associated with complications. We present the first reported case of a colojejunal fistula resulting from a D-PEJ placement. While D-PEJ has been shown to be relatively safe, complications related to the inherent limitations of the procedure need to be considered when the patient experiences unusual post-procedure symptoms and worked up appropriately.
\end{abstract}

\section{Case Report}

A 40-year-old woman with a 30-year history of type 1 diabetes mellitus complicated by gastroparesis necessitating long-term enteral nutrition complained of three weeks of worsening abdominal pain and severe diarrhea. Two years previously, she had undergone an outpatient D-PEJ placement for enteral access due to severe gastroparesis. At the time of the procedure, transillumination of the light was noted in the left mid abdomen. The tube was placed without any difficulty using a modified Seldinger technique. No perioperative antibiotics were administered. The patient was evaluated in the recovery area and dismissed home. The night of the procedure she experienced significant abdominal pain around the D-PEJ site. Her primary physician saw her the next day. An abdominal/pelvic CT scan was ordered which revealed a small amount of free air without free fluid (fig. 1). The jejunal tube site was closely adhered to the abdominal wall. Her pain improved over the following 24 hours and she returned home. While at home, she experienced significant crampy abdominal pain with each feeding, persistent sharp pain in the abdomen under the tube insertion site and recurrent skin cellulitis near the tube. She was followed by a multidisciplinary enteral nutrition team which had placed her on antibiotics when needed. 
One year after placement, she had the long feeding tube replaced under endoscopic visualization to a feeding button with a $5 \mathrm{~cm}^{3}$ anchoring balloon. At the time of endoscopy, a small bowel 'diverticulum' was noted in the bowel wall just opposite the tube insertion site. She continued to have persistent symptoms related to her feeding apparatus despite the tube exchange. Because of persistent pain associated with the tube site, she was referred to a chronic pain clinic. After failed trials of medications she underwent percutaneous celiac nerve blocks twice without significant improvement in her symptoms. Three weeks prior to presentation, the patient noted worsening of her pain around the feeding tube site. This was accompanied by low-grade fevers but no other signs of local infection or systemic toxicity. She also noted the onset of severe diarrhea that was associated with initiating her daily feedings. By her report, the diarrhea would begin within minutes of feeding and looked similar to her tube feed formula. She was seen by her primary physician. On exam, she was afebrile, hemodynamically stable and had tenderness around the feeding button site but no evidence of peritonitis. A CT scan with oral contrast was obtained that revealed no free air, no free abdominal fluid and no extravasation of contrast but was concerning for the feeding tube balloon being in the colon rather in the small bowel. An exploratory celiotomy was recommended, but the patient requested transfer to another institution for evaluation.

On transfer, the patient appeared tired but in no acute distress. Her exam was notable for tenderness around the feeding tube site but no guarding or peritoneal signs. She was afebrile with mild tachycardia and hypertension but was otherwise stable. Her laboratory exam revealed a white blood cell count of 3.8 and an albumin of 4.2. A sinogram with water-soluble contrast through the feeding button demonstrated free flow into the small intestine without any obvious communication with the colon. Later, a CT sinogram was obtained that demonstrated flow directly into the colon with visualization of

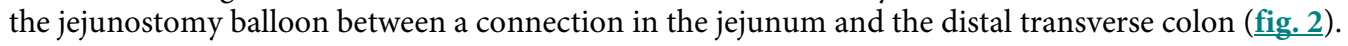

After discussion with the patient, an exploratory celiotomy was recommended. She was placed in combined position with an abdominal and perineal prep. An upper midline incision was made to just above the umbilicus. Upon entry there was no evidence of ascites or purulence. Inflammation was minimal. The jejunum at the site of the feeding tube was brought into view. The distal transverse colon was densely adherent to the medial aspect of the loop of jejunum were the feeding tube was placed. The balloon of the feeding button was easily palpated and could be pushed into the colon or the small bowel with minimal effort. When placed in one lumen, the balloon occluded the fistulous communication to the opposite lumen (fig. 3). The balloon was deflated and the device was removed. The fistula tract was resected with primary repair of both small and large intestine. A new feeding jejunostomy was placed distal to the small bowel repair.

The patient did well after the procedure. She was started on her home tube feeding regimen. She was discharged home on postoperative day 5 without further episodes of diarrhea or abdominal pain. Follow-up four weeks later revealed that her abdominal wall pain and crampy pain with feeding had completely resolved.

\section{Discussion}

Placement of chronic enteral feeding devices has markedly improved the care of patients who require nutritional support. Originally, placement of these devices required surgical placement through a laparotomy incision. The development of endoscopic techniques for placement has dramatically decreased the morbidity, inconvenience, and cost of the procedure. There are numerous endoscopic procedures with D-PEJ being the newest among these. D-PEJ was first described by Ponsky and Adzodi in 1984 [1]. It was developed to meet the needs of patients with proximal disease after unsatisfactory results from PEG-J [2-5]. The technique involves push enteroscopy utilizing a long endoscope advanced into the jejunum. Light is transilluminated through the anterior abdominal wall in a distinct spot. Manipulation of the jejunum is sometimes necessary in order to obtain this clear light point. A needle placed into an incision immediately above the light is placed into the jejunum under direct endoscopic visualization. A guide wire is cannulated through the needle. The snare of the endoscope grabs the wire and is withdrawn from the patient's mouth as the scope is removed. The feeding tube is secured to the wire and the tube is pulled into position by pulling on the wire at the skin exit site on the abdominal 
wall. Proper placement in the jejunum is confirmed by endoscopic visualization and the device is secured tightly to the abdominal wall in order to prevent the bowel from falling.

While feeding tube procedures are relatively straightforward, complications such as occlusion, malposition and peritube leakage are not uncommon $[1,3-6]$. The critical portion of the D-PEJ involves transillumination. Distinct light transmission is a necessity prior to needle catheterization of the jejunum. If the operator is unable to see the transillumated light, the endoscopic procedure should be aborted. Another endoscopic technique, as some institutions have suggested, is to perform the D-PEJ jointly with fluoroscopy [6]. Combination of these techniques allows for visualization of other intraperitoneal structures, most importantly the transverse colon. However, equivalent rates of complications have been reported for PEG, PEG-J and D-PEJ placed fluoroscopically versus purely endoscopic placement [7]. Lastly, surgical placement of a feeding jejunostomy via laparoscopic or mini-laparotomy techniques is available. These methods are perhaps the safest in respect to ensuring proper tube placement but are not without their own set of complications, risks to the patient, and involve greater expense [8]. Operative feeding tube placement should be considered the procedure of choice in those patients who have had a history of prior major abdominal surgery as adhesion formation may have altered the normal intestinal anatomy, making endoscopic placement more difficult.

Gastrocolocutaneous fistula formation from PEG placement has been described but we report the first case of a colojejunal fistula resulting from a D-PEJ tube $[9,10]$. Our impression, based on the history and operative findings, was that this patient had a bowel perforation with a small leak on the opposite wall of the intestine at the time of her initial feeding tube placement. Although these findings can be normal [11], this would account for her pain the night of the procedure and the CT scan finding of free air. This small leak was subsequently sealed by the transverse colon. Over time, a small fistulous communication developed that was noted on endoscopy one year later as the small bowel 'diverticulum.' The balloon anchor from the new feeding button slowly eroded the fistulous communication open further resulting in her symptoms related to the feeding device. These symptoms, and the absence of demonstrating the fistula on the sinogram, can be explained from intermittent obstruction of the fistula based on the location of the button's balloon. Another theory could be postulated that the colon was traversed during the initial placement. The subsequent tube exchange may have led to the passage of the feeding tube into the colon, with subsequent intermittent obstruction of the fistula tract.

Endoscopic placement of enteral feeding devices represents a significant improvement in care for patients who require them. It has reduced the morbidity, inconvenience, and cost to the patient as compared to operatively placed devices. Given the inherent limitations of the technique, a number of complications have been reported, but this is the first reported case of a colojejunal fistula after D-PEJ placement. Although the procedure was performed without any breaks in technique, the patient's unusual post-procedure course should have raised the possibility of a device-related complication. Although the addition of fluoroscopy may be helpful in some patients, it is unclear whether this would have been useful in this case, as she had no prior major abdominal operations. We do not routinely require post-procedure inpatient hospitalization if the patient is otherwise doing well after several hours of observation in the recovery area, although this would have facilitated earlier evaluation of her severe pain and likely not changed her overall outcome. While this is an extremely unusual and likely unavoidable complication after an endoscopic placement of an enteral access device, the chronic nature of the patient's 
post-procedure symptoms should raise the concern that the device could have been the cause of her difficulty and should have been thoroughly investigated.

Fig. 1. Initial post-procedure $\mathrm{CT}$ scan.

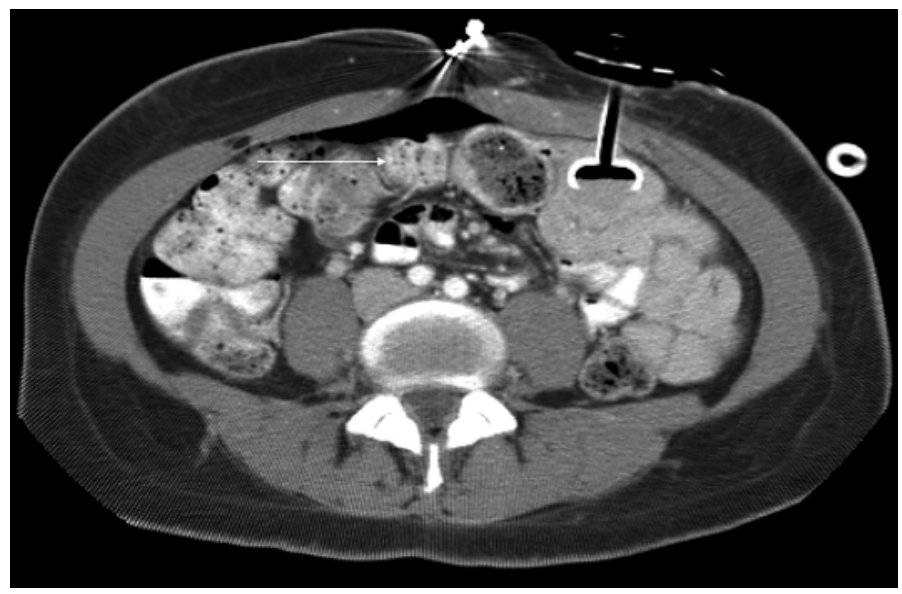

Fig. 2. CT sinogram of colojejunal fistula.

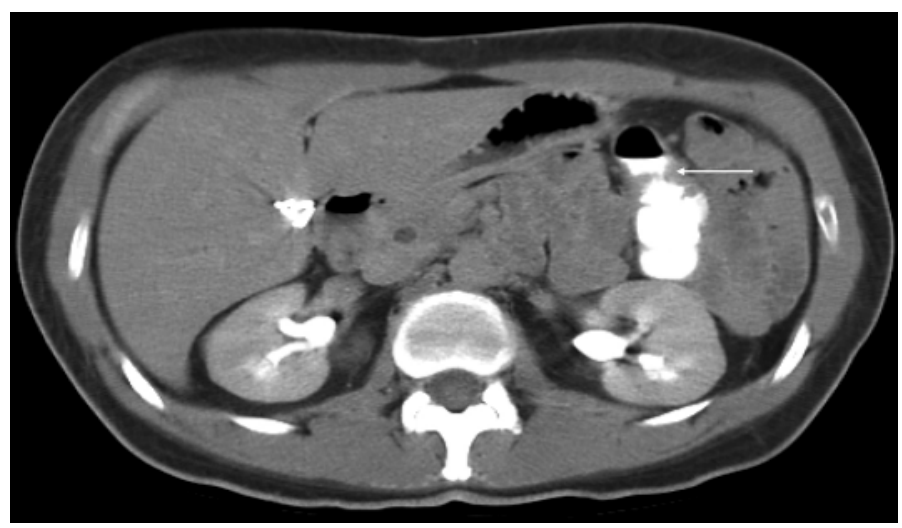


Case Reports in | Case Rep Gastroenterol 2008;2:208-213 Dol: $10.1159 / 000136018$

Fig. 3. Intraoperative picture of colojejunal fistula.

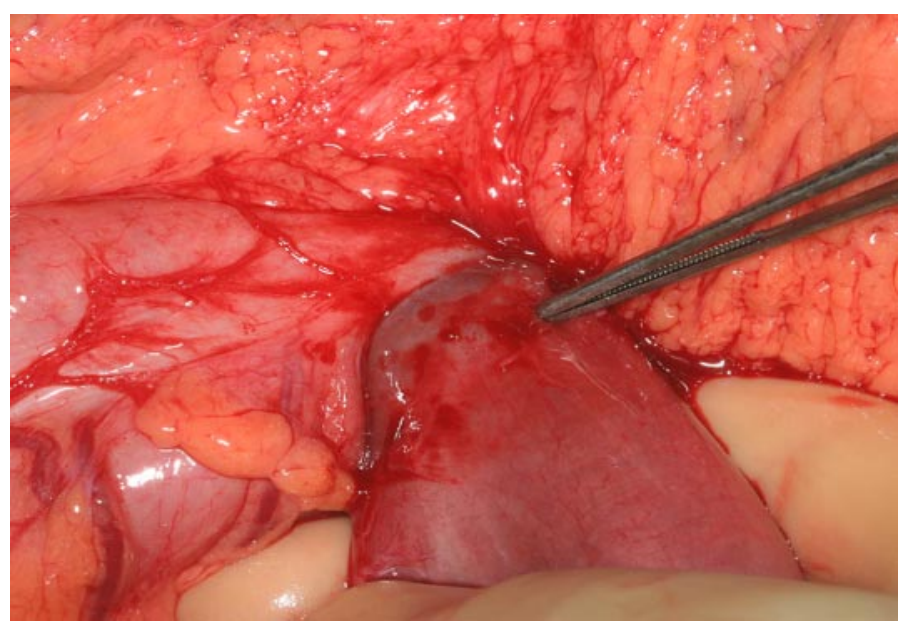




\section{References}

1 Ponsky JL, Aszodi A: Percutaneous endoscopic jejunostomy. Am J Gastroenterol 1984;79:113-116.

2 Fan AC, Baron TH, Rumalla A, et al: Comparison of direct percutaneous endoscopic jejunostomy and PEG with jejunal extension. Gastrointest Endosc 2002;56:890-894.

-3 Henderson JM, Strodel WE, Gilinsky NH: Limitations of percutaneous endoscopic jejunostomy. JPEN J Parenter Enteral Nutr 1993;17:546-550.

4 Disario JA, Foutch PG, Sanowski RA: Poor results with percutaneous endoscopic jejunostomy. Gastrointest Endosc 1990;36:257-260.

-5 Wolfsen HC, Kozarek RA, Ball TJ, et al: Tube dysfunction following percutaneous endoscopic gastrostomy and jejunostomy. Gastrointest Endosc 1990;36:261-263.

6 Shetzline MA, Suhocki PV, Worman MJ: Direct percutaneous endoscopic jejunostomy with small bowel enteroscopy and fluoroscopy. Gastrointest Endosc 2001;53:633-638.

7 Cope C, Davis AG, Baum RA, et al: Direct percutaneous jejunostomy: Techniques and applications - ten years experience. Radiology 1998;209:747-754.

8 Holmes JH 4th, Brundage SI, Yuen, et al: Complications of surgical feeding jejunostomy in trauma patients. J Trauma 1999;47:1009-1012.

9 Smith SE, Clancy TV: Colocutaneous fistula. A rare complication of percutaneous gastrostomy. N C Med J 1998;59:80-82.

10 Yamazaki T, Sakai Y, Hatakeyama K: Colocutaneous fistula after percutaneous endoscopic gastrostomy in a remnant stomach. Surg Endosc 1999;13:280-282.

11 Wojotowycz MM, Arata JA, Micklos TJ, et al: CT findings after uncomplicated percutaneous gastrostomy. Am J Roentgenol 1988;151:307-309. 